\title{
The Diagnostic Yield and Cost of Radiologic Imaging for Urgent and Emergent Ocular Conditions in an Eye Emergency Room
}

\author{
Jordan D. Deaner, MD ${ }^{1}$ Austin R. Meeker, MD ${ }^{1}$ Daniel J. Ozzello, MD ${ }^{1} \quad$ Vishal Swaminathan, BS ${ }^{1}$ \\ Dilru C. Amarasekera, MD ${ }^{1}$ Qiang Zhang, PhD $^{2}$ Rose A. Hamershock, MA ${ }^{2}$ Robert C. Sergott, MD ${ }^{1}$ \\ ${ }^{1}$ Neuro-Ophthalmology Service, Wills Eye Hospital, Philadelphia, \\ Pennsylvania \\ 2 Biostatistics Consulting Core, Vicky and Jack Farber Vision Research \\ Center, Wills Eye Hospital, Philadelphia, Philadelphia \\ Address for correspondence Robert C. Sergott, MD, Neuro- \\ Ophthalmology Service, Wills Eye Hospital, 840 Walnut Street, \\ Philadelphia, PA 19107 (e-mail: rcs220@comcast.net). \\ J Acad Ophthalmol 2020;12:e115-e123.
}

\begin{abstract}
Keywords

- diagnostic yield

- imaging

- cost

- economic

- emergency room

Objective The aim of this study is to assess the diagnostic yield and economic cost of radiologic imaging for urgent and emergent ophthalmic conditions in an emergency room (ER) setting

Design Retrospective, consecutive case series.

Methods Charts of all patients who underwent radiologic imaging in a dedicated eye ER over a single year were reviewed. Data collected included age, patient reported chief complaint, visual acuity, principal examination finding, indication for imaging, imaging modalities performed, and the current procedural terminology (CPT) codes billed for the imaging performed. Imaging results were classified into three groups with binary outcomes: normal or abnormal; significant if it led to a change in patient management, and relevant if the imaging findings were related to the chief complaint or principal examination finding. Imaging costs were calculated using the billed CPT codes.

Results A total of 14,961 patients were evaluated during the 1-year study and 1,371 (9.2\%) patients underwent imaging. Of these, 521 patients $(38.0 \%)$ had significant findings. A majority of this group had significant and relevant findings (469, 34.2\% of total). Subgroup analysis was performed based upon patient chief complaint, principal examination finding, and indication for imaging. Overall, the total cost of imaging was $\$ 656,078.34$ with an average cost of $\$ 478.54$ per patient.

Conclusion Imaging for urgent and emergent ophthalmic conditions in an eye ER resulted in significant management changes in $38.0 \%$ of patients. Radiographic imaging contributes to healthcare expenditures; however, these costs must be weighed against the substantial costs of delayed and misdiagnoses, especially when patients present with acute ophthalmological symptoms.
\end{abstract}

Diagnostic imaging has revolutionized the practice of ophthalmology. Computerized tomographic (CT) and magnetic resonance imaging (MRI) have empowered ophthalmologists to diagnose many ophthalmic orbital and neuro-ophthalmic conditions that previously escaped immediate diagnosis. In an era fraught with medical malpractice and pressure to practice cost-effective medicine, the goal of most physicians is to order studies only when results (either positive or negative) will lead to the diagnosis or a change in patient management. ${ }^{1}$ Otherwise, inappropriate testing, excessive received

May 18,2020

accepted

July 7, 2020
DOI https://doi.org/

10.1055/s-0040-1715570. ISSN 2475-4757.
Copyright $\odot 2020$ by Thieme Medical Publishers, Inc., 333 Seventh Avenue, New York, NY 10001, USA. Tel: +1(212) 760-0888.
License terms

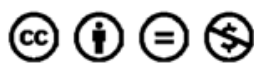


testing, or lack of needed testing may incur a substantial cost to our healthcare system. ${ }^{1}$ Overutilization of imaging has been a topic of particular concern in United States emergency departments (ED), ${ }^{2-6}$ where increasing utilization of diagnostic imaging leads to not only increased costs, ${ }^{7}$ but also prolonged ED lengths of stay. ${ }^{8}$

Previous studies have evaluated the utilization and yield of radiologic imaging in the outpatient work-up of neuroophthalmic $^{9-15}$ and orbital disease. ${ }^{16,17}$ Others have assessed the yield of radiologic imaging in the setting of ocular trauma. ${ }^{18-20}$ We sought to describe the diagnostic yield and cost of radiologic imaging for urgent and emergent ocular conditions in a dedicated eye emergency room (ER).

\section{Methods}

The Wills Eye Hospital Institutional Review Board approved this study and the clinical research complied with the Declaration of Helsinki. Informed consent was not obtained in this retrospective, noninterventional, consecutive case series. Our study included all patients evaluated in the Wills Eye Emergency Room over a 1-year period (April 1, 2017 to March 31, 2018) who underwent CT or MRI imaging of the head, orbits, face, skull base, neck, and associated vasculature. When multiple imaging studies were performed (i.e., MRI brain and orbits) each modality was recorded, but their results were analyzed as a single imaging study. Patients were excluded if they were referred from an outside institution with prior imaging.

Data collected included age, visual acuity (VA), patientreported chief complaint (CC), principal examination finding, indication for imaging, the type and number of imaging modalities performed, and the current procedural terminology (CPT) codes that were billed for the imaging performed. Imaging findings were categorized into three groups with binary outcomes: normal versus abnormal, significant versus nonsignificant, and relevant versus nonrelevant. A finding was defined as significant if it prompted a change in patient management. A finding was determined to be relevant if it accounted for the patient's CC or principal examination finding.

Diagnostic yield was defined as the percentage of patients with a significant imaging finding which prompted a change in patient care. Results were then organized into 1 of 6 outcome groups based upon their categorical binary outcomes for more granular analysis: (1) abnormal, significant, relevant, (2) abnormal, significant, nonrelevant, (3) abnormal, nonsignificant, relevant, (4) abnormal, nonsignificant, nonrelevant, (5) normal, significant, nonrelevant, or (6) normal (nonsignificant, nonrelevant).

Exploratory subgroup analysis was performed based upon patient-reported CC, principal examination finding, and indication for imaging. A full list of CC is reported in -Table $\mathbf{1}$. Visual disturbance was defined as those patients who complained of a subjective change in visual perception but did not experience subjective decrease in VA or fit into another category. The principal examination finding was defined as the examination finding that prompted imaging. If a patient had more than one examination finding, the examination finding that most strongly prompted imaging was selected.
A full list of principal examination findings is reported in - Table 2. The principal examination finding of "decreased vision" was only utilized if the patient had a VA worse than 20/20 and no other examination findings. Indication for imaging is synonymous with the suspected diagnosis that the physician entered upon ordering the imaging test, prior to imaging results ( - Table 3 ).

The cost of imaging was determined using the Physician Fee Schedule Search found at the Centers of Medicare and Medicaid Services website and the 2018 conversion factor. ${ }^{21}$ Although all CPT codes from each visit were collected, only the billed CPT codes were analyzed. Thus, if an MRI brain and orbits were performed sequentially, only the higher cost CPT code was analyzed in the cost analysis. The professional and technical components were combined to obtain the total cost of the individual imaging studies (-Table 4). No regional adjustments were performed.

All statistical analyses were performedby Q.Z. and R.A.H. using SAS 9.4 (SAS Institute, Inc, Cary, NC). The overall yield rate and its $95 \%$ confidence intervals (CIs) were calculated. Exploratory subgroup analysis was performed as described above. The costs of imaging per outcome group and per significant finding was calculated. Comparisons between imaging outcome groups were tested with the KruskalWallis test for continuous variables and the Fisher's Exact test with Monte Carlo estimate for categorical variables. Comparisons between significant outcome groups were evaluated with the chi-square test or with Fisher's exact test when the expected cell count was $<5$ for categorical variables with a Monte Carlo estimate, when appropriate. Continuous variables were compared with the Rank Sum test. The Goodman method with Bonferroni adjustment was used to compute simultaneous CI. A two-sided $\alpha$ level of 0.05 was used to determine significance.

\section{Results}

From April 1, 2017 to March 31, 2018, 14,961 patients presented to the Wills Emergency Room for evaluation. Of those patients, 1,371 (9.2\%) met inclusion criteria and underwent imaging based upon their presentation and examination. A majority of patients had MRI imaging studies performed ( 880 , $64.2 \%$ ). The average age was 47 years, ranging from 5 to 97 years. In total, 2,703 imaging studies were obtained over the year. The affected eye(s) was more often unilateral (36\% right eye, 35\% left eye), than bilateral (29\%).

Of the 1,371 patients who underwent imaging, 521 (38.0\%) had significant findings that resulted in a change in patient management (- Table 5). Most of this group (469, 34.2\%) had significant and relevant findings. Thirty-six patients $(2.6 \%)$ had incidental, significant, but not relevant findings on imaging. An example from this outcome group was a patient with an incidentally discovered anterior communicating artery aneurysm unrelated to their symptoms and examination, but requiring neurosurgical evaluation. Finally, of the significant groups, 16 patients (1.2\%) had normal, significant, but nonrelevant exams. This group consisted solely of patients diagnosed with idiopathic intracranial hypertension with normal scans 


\begin{tabular}{|c|c|c|c|c|c|c|c|c|c|c|c|c|c|c|c|c|c|c|c|}
\hline & 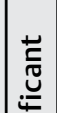 & 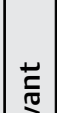 & $\stackrel{\circ}{\circ}$ & $\begin{array}{l}0 \\
\infty \\
\infty\end{array}$ & $\frac{\widehat{\sigma}}{\grave{d}}$ & $\begin{array}{l}\widehat{m} \\
\grave{\omega} \\
\underline{v}\end{array}$ & 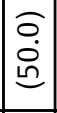 & $\begin{array}{l}\bar{m} \\
\stackrel{p}{\Xi}\end{array}$ & 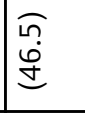 & 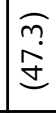 & $\begin{array}{l}\sigma \\
\infty \\
\infty \\
\tilde{c}\end{array}$ & \begin{tabular}{|l}
$\hat{O}$ \\
$\dot{m}$ \\
$\dot{y}$
\end{tabular} & $\begin{array}{l}\infty \\
\dot{\infty} \\
\tilde{c} \\
\mid\end{array}$ & \begin{tabular}{|l}
0 \\
$\dot{0}$ \\
$\dot{0}$ \\
0
\end{tabular} & 令 & $\begin{array}{l}\underset{\sigma}{d} \\
\underset{d}{d}\end{array}$ & $\begin{array}{l}\widehat{\hat{\sigma}} \\
\dot{b} \\
\dot{b}\end{array}$ & $\begin{array}{l}0 \\
\dot{0} \\
\dot{0}\end{array}$ & $\begin{array}{l}\widehat{m} \\
\stackrel{d}{\varrho} \\
\stackrel{d}{n}\end{array}$ \\
\hline $\begin{array}{l}\frac{\pi}{5} \\
\vdots \\
2\end{array}$ & $\begin{array}{c}\overline{\overline{5}} \\
\bar{n} \\
1\end{array}$ & $\left|\begin{array}{c}\frac{\bar{u}}{\bar{\alpha}} \\
1\end{array}\right|$ & $=$ & $\infty$ & $m$ & $\stackrel{+}{\circ}$ & $N$ & - & $\stackrel{\sim}{N}$ & $\stackrel{\varrho}{\sim}$ & 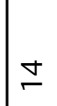 & नू & $\dot{m}$ & 0 & $\mp$ & in & 0 & $\curvearrowright$ & in \\
\hline$\overline{\widetilde{\pi}}$ & 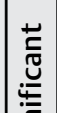 & 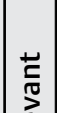 & $\widehat{\triangleright}$ & $\mid \begin{array}{c}\widehat{a} \\
\\
\end{array}$ & $\begin{array}{l}\bar{m} \\
\stackrel{+}{\Xi}\end{array}$ & $\begin{array}{l}\widehat{a} \\
\dot{0} \\
=\end{array}$ & $\begin{array}{l}o \\
\dot{e}\end{array}$ & 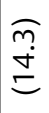 & ơ & $\begin{array}{l}\widehat{\sigma} \\
\ddot{n}\end{array}$ & $\begin{array}{l}\widehat{\widetilde{N}} \\
\stackrel{\mathbb{N}}{\boldsymbol{N}}\end{array}$ & 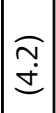 & $\stackrel{\check{c}}{\overline{\grave{d}}}$ & 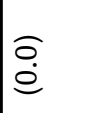 & $\stackrel{\Im}{\stackrel{f}{\leftrightarrows}}$ & 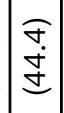 & 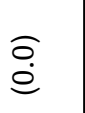 & 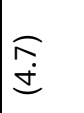 & $\begin{array}{l}\widehat{\infty} \\
\dot{0} \\
\stackrel{0}{0}\end{array}$ \\
\hline $\begin{array}{l}\frac{5}{<} \\
\frac{1}{<}\end{array}$ & $\begin{array}{c}\bar{n} \\
1\end{array}$ & $\begin{array}{l}\widetilde{\widetilde{x}} \\
+\end{array}$ & $=$ & $\stackrel{2}{N}$ & $\sim$ & $\stackrel{q}{q}$ & 0 & - & 0 & $\sim$ & $\infty$ & a & $\stackrel{\sim}{\sim}$ & 0 & $\sim$ & の & 0 & 6 & $r$ \\
\hline$\overline{\widetilde{\pi}}$ & 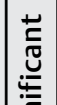 & $\mid \begin{array}{l}\overrightarrow{\mathrm{T}} \\
\mathrm{T} \\
\mathrm{v}\end{array}$ & $\stackrel{\bigcirc}{\circ}$ & 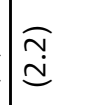 & 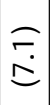 & 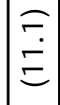 & $\begin{array}{l}0 \\
\dot{0}\end{array}$ & $\begin{array}{l}\widetilde{\sigma} \\
\stackrel{\infty}{\simeq} \\
\stackrel{0}{v}\end{array}$ & 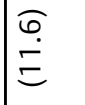 & $\stackrel{\bar{\sigma}}{\dot{\sigma}}$ & $\begin{array}{l}\widehat{\infty} \\
\stackrel{d}{c}\end{array}$ & $\begin{array}{l}0 \\
\dot{0} \\
=\end{array}$ & 命 & 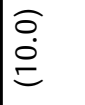 & $\begin{array}{l}\widetilde{N} \\
\stackrel{N}{v}\end{array}$ & $\begin{array}{l}0 \\
0 \\
0 \\
0\end{array}$ & 咅 & $\stackrel{\substack{n \\
ٍ}}{\varrho}$ & 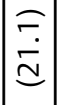 \\
\hline $\begin{array}{l}\text { 妾 } \\
\end{array}$ & $\begin{array}{l}n \\
1\end{array}$ & $\begin{array}{c}\simeq \\
1\end{array}$ & $=$ & $\sim$ & - & Ұ & 0 & $\sim$ & in & in & - & $\stackrel{\infty}{m}$ & $a$ & - & 6 & $\stackrel{\Xi}{\sim}$ & - & $\approx$ & $\nabla$ \\
\hline & 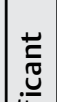 & & ঐे & 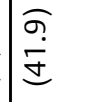 & $\begin{array}{l}\widehat{\sigma} \\
\stackrel{ \pm}{ \pm}\end{array}$ & 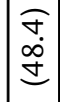 & \begin{tabular}{|l|}
0 \\
0 \\
$\dot{0}$ \\
\end{tabular} & 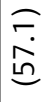 & 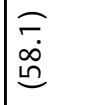 & $\begin{array}{l}0 \\
\dot{0} \\
\dot{0} \\
0\end{array}$ & $\begin{array}{l}\hat{\sigma} \\
\hat{\tilde{\theta}}\end{array}$ & $\begin{array}{l}0 \\
\dot{j} \\
\dot{f} \\
\end{array}$ & \begin{tabular}{|l}
$\hat{m}$ \\
$\dot{\theta}$ \\
$\dot{e}$
\end{tabular} & 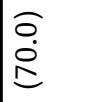 & f̊ & $\begin{array}{l}0 \\
\stackrel{0}{i} \\
\vdots\end{array}$ & $\begin{array}{l}\widehat{\infty} \\
\stackrel{1}{\Sigma} \\
\stackrel{5}{\Sigma}\end{array}$ & \begin{tabular}{|l}
$\hat{\widehat{N}}$ \\
$\infty$ \\
$\infty$ \\
0
\end{tabular} & $\begin{array}{l}\text { ָิ } \\
\dot{+} \\
\dot{\infty}\end{array}$ \\
\hline & $\bar{n}$ & & $=$ & $\stackrel{m}{m}$ & 0 & $\bar{\sigma}$ & $N$ & $\nabla$ & 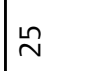 & $\stackrel{m}{m}$ & $\stackrel{n}{\sim}$ & $\underline{m}$ & $\hat{\sigma}$ & $r$ & $\stackrel{9}{-}$ & $\stackrel{m}{\stackrel{n}{=}}$ & $\wedge$ & $\stackrel{\text { 옹 }}{\circ}$ & $\stackrel{0}{\circ}$ \\
\hline & 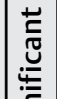 & 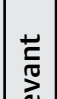 & ङ̊ & $\mid \begin{array}{c}0 \\
\dot{e}\end{array}$ & 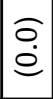 & 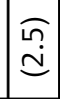 & ộ & ọ & ọ & 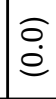 & 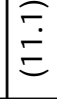 & 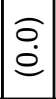 & $\stackrel{\check{C}}{\check{\Xi}}$ & 官 & $\stackrel{o}{0}$ & $\stackrel{o}{\dot{0}}$ & $\begin{array}{l}\text { ọ } \\
\stackrel{\varrho}{\varrho}\end{array}$ & 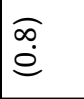 & ô. \\
\hline $\bar{z}$ & $\begin{array}{l}n \\
+\end{array}$ & 1 & $=$ & 0 & 0 & $\circ$ & 0 & 0 & 0 & 0 & $\nabla$ & 0 & - & 0 & 0 & 0 & 0 & - & 0 \\
\hline$\overline{\widetilde{\pi}}$ & 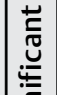 & $\begin{array}{l}\overrightarrow{\mathrm{N}} \\
\mathrm{N} \\
\mathrm{T}\end{array}$ & ङ̊ & 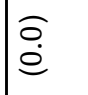 & ọ & 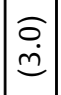 & $\begin{array}{l}a \\
\stackrel{0}{e}\end{array}$ & ọ & 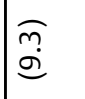 & $\stackrel{\partial}{\stackrel{0}{e}}$ & $\stackrel{\widehat{\infty}}{\stackrel{d}{\mathfrak{d}}}$ & $\begin{array}{l}a \\
\dot{d}\end{array}$ & $\stackrel{\overrightarrow{\check{c}}}{\underline{\underline{c}}}$ & $\begin{array}{l}\stackrel{0}{0} \\
\stackrel{0}{=}\end{array}$ & 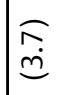 & $\stackrel{\bar{m}}{=}$ & $\begin{array}{l}\text { ọ } \\
\stackrel{e}{e}\end{array}$ & 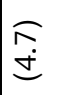 & 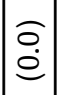 \\
\hline ₹ & + & & $=$ & 0 & 0 & $\simeq$ & 10 & 0 & $\nabla$ & 0 & - & 6 & $\sim$ & - & - & $m$ & 0 & 0 & 0 \\
\hline $\bar{c}$ & 节 & $\begin{array}{l}\vec{U} \\
\text { 节 }\end{array}$ & ঐ & 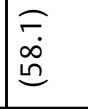 & $\begin{array}{l}\tilde{E} \\
\text { in }\end{array}$ & 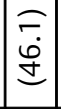 & \begin{tabular}{|c|}
0 \\
0 \\
$\dot{0}$ \\
\end{tabular} & $\begin{array}{l}\widehat{a} \\
\stackrel{\Xi}{ \pm}\end{array}$ & $\begin{array}{l}\hat{\sigma} \\
\stackrel{i}{\sim}\end{array}$ & 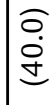 & $\begin{array}{l}\tilde{N} \\
\stackrel{\mathbb{N}}{v}\end{array}$ & $\mid \begin{array}{l}\tilde{N} \\
\tilde{n} \\
\tilde{c}\end{array}$ & 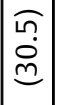 & ộ & $\begin{array}{l}\widehat{a} \\
\stackrel{\mathfrak{d}}{\beth}\end{array}$ & $\stackrel{\overline{\check{c}}}{\overline{\grave{c}}}$ & 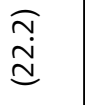 & $\begin{array}{l}\hat{\infty} \\
\stackrel{\bar{E}}{E}\end{array}$ & $\begin{array}{l}\hat{\infty} \\
\stackrel{1}{\rho} \\
=\end{array}$ \\
\hline 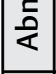 & $\begin{array}{l}\bar{n} \\
+\end{array}$ & $\begin{array}{l}\widetilde{\simeq} \\
+\end{array}$ & $=$ & เ̂' & $\infty$ & $\underset{\infty}{\infty}$ & $\sim$ & $m$ & \pm & $\approx$ & $\infty$ & $\pi$ & $\stackrel{\text { D }}{2}$ & $\sim$ & $\wedge$ & f & $\sim$ & $\stackrel{\llcorner}{\leftarrow}$ & $m$ \\
\hline & 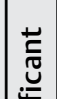 & & ๖े & $\mid \begin{array}{c}\bar{\rho} \\
\infty \\
\stackrel{\infty}{n}\end{array}$ & $\begin{array}{l}\tilde{r} \\
\hat{i}\end{array}$ & \begin{tabular}{|l|}
0 \\
$\dot{1}$ \\
$\underline{n}$
\end{tabular} & \begin{tabular}{|l|}
0 \\
0 \\
$\dot{0}$ \\
\end{tabular} & $\begin{array}{l}\widehat{\sigma} \\
\stackrel{\text { I}}{ \pm}\end{array}$ & $\begin{array}{l}\widehat{\sigma} \\
\frac{\sigma}{\dot{y}}\end{array}$ & $\begin{array}{l}\text { oे } \\
\dot{+}\end{array}$ & $\begin{array}{l}\overline{\bar{\varphi}} \\
\dot{0}\end{array}$ & $\mid \begin{array}{l}0 \\
\dot{0} \\
0 \\
0\end{array}$ & $\begin{array}{l}\hat{R} \\
\dot{m} \\
\tilde{c}\end{array}$ & $\begin{array}{l}a \\
\dot{0} \\
\dot{n}\end{array}$ & 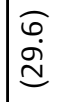 & 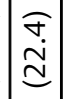 & 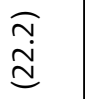 & 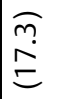 & 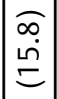 \\
\hline & $\begin{array}{l}n \\
+\end{array}$ & & $=$ & 点 & $\infty$ & 定 & $\sim$ & $m$ & $\stackrel{\infty}{\sim}$ & $\approx$ & $m$ & $\curvearrowright$ & $\tilde{m}$ & $m$ & $\infty$ & in & $\sim$ & $\approx$ & $m$ \\
\hline & & & 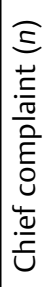 & 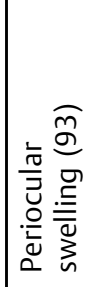 & 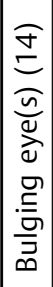 & 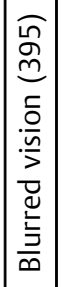 & 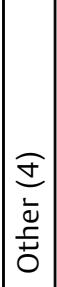 & 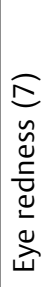 & 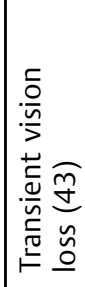 & 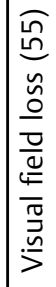 & 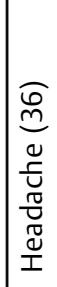 & 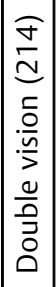 & 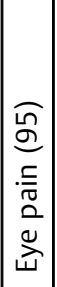 & 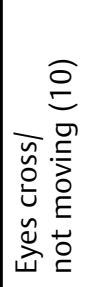 & 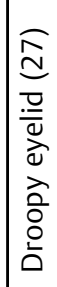 & 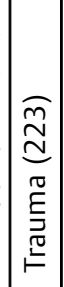 & 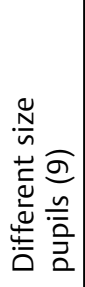 & 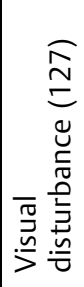 & 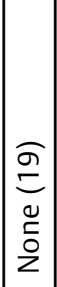 \\
\hline
\end{tabular}




\begin{tabular}{|c|c|c|c|c|c|c|c|c|c|c|c|c|c|c|c|c|c|c|c|c|c|c|c|}
\hline & 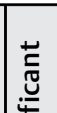 & $\overrightarrow{\mathrm{v}}$ & ळ & 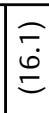 & $\begin{array}{l}\sigma \underline{0} \\
\dot{\Xi} \\
=\end{array}$ & 尽 & $\mid \begin{array}{c}\tilde{m} \\
\stackrel{n}{v}\end{array}$ & 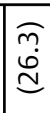 & 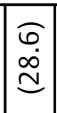 & $\begin{array}{l}\widehat{\infty} \\
\dot{\varphi}\end{array}$ & 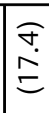 & $\begin{array}{l}\hat{\partial} \\
\dot{\rho} \\
\stackrel{0}{0}\end{array}$ & 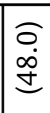 & 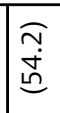 & $\mid \begin{array}{r}\tilde{\imath} \\
\dot{d} \\
\grave{d}\end{array}$ & \begin{tabular}{|l}
$\widehat{m}$ \\
$\stackrel{\rho}{\cong}$ \\
$\stackrel{n}{n}$
\end{tabular} & 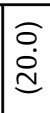 & $\begin{array}{l}\text { 年 } \\
\stackrel{\text { fo }}{y}\end{array}$ & $\mid \begin{array}{l}\widehat{T} \\
\dot{\theta} \\
0\end{array}$ & 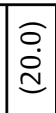 & 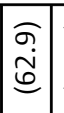 & $\begin{array}{l}\bar{F} \\
\Xi \\
\Xi\end{array}$ & $\begin{array}{l}\text { ô. } \\
\text { in }\end{array}$ \\
\hline $\begin{array}{l}\tilde{E} \\
\text { है }\end{array}$ & 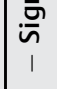 & 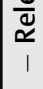 & $=$ & $\sigma$ & 6 & 6 & $m$ & $\curvearrowright$ & 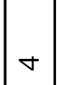 & 0 & $\simeq$ & $\tilde{\sigma}$ & $\simeq$ & $\stackrel{\mathscr{N}}{\sim}$ & F & 6 & - & in & $\stackrel{\stackrel{n}{口}}{=}$ & $\mp$ & \& & - & เn \\
\hline & 节 & 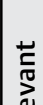 & ஓे & $\mid \begin{array}{l}\widehat{\sigma} \\
\infty\end{array}$ & 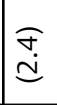 & $\begin{array}{l}\infty \\
\stackrel{\infty}{=} \\
=\end{array}$ & ב்َ & $\begin{array}{l}\tilde{\partial} \\
\dot{\dot{\Xi}} \\
=\end{array}$ & 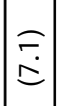 & $\mid \begin{array}{l}\infty \\
\dot{\rho} \\
\tilde{e}\end{array}$ & $\mid \begin{array}{l}\infty \\
\infty \\
= \\
=\end{array}$ & 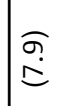 & $\widehat{a}$ & $\begin{array}{l}\bar{m} \\
\infty\end{array}$ & $\mid \begin{array}{c}\widehat{̃} \\
\stackrel{0}{0} \\
\end{array}$ & 官 & \begin{tabular}{|l}
0 \\
$\dot{0}$ \\
$\dot{0}$
\end{tabular} & 官 & ô & $\begin{array}{l}0 \\
\dot{0} \\
\dot{0}\end{array}$ & $\stackrel{\widehat{m}}{\dot{ \pm}}$ & $\begin{array}{l}\widehat{\infty} \\
\stackrel{\Sigma}{\Sigma}\end{array}$ & $\begin{array}{l}\text { ○े } \\
\stackrel{0}{\circ}\end{array}$ \\
\hline & & & $=$ & & - & 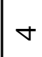 & $r$ & $\bar{\gamma}$ & - & $\stackrel{\llcorner}{m}$ & $\stackrel{m}{\sim}$ & $\nexists$ & 0 & $\theta$ & $\widetilde{\sigma}$ & 0 & $m$ & 0 & 0 & $m$ & $m$ & $\wedge$ & - \\
\hline
\end{tabular}

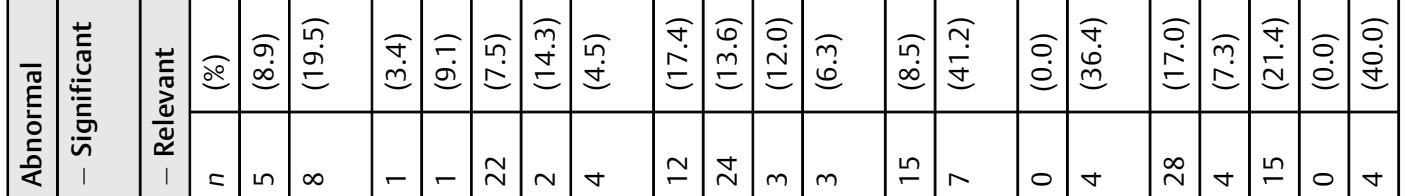

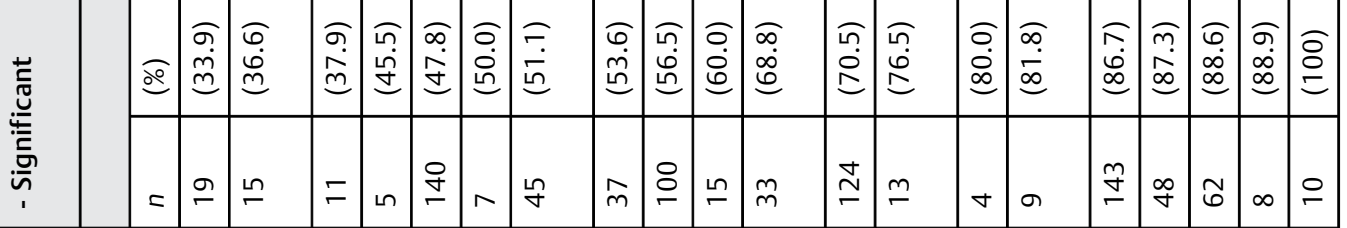

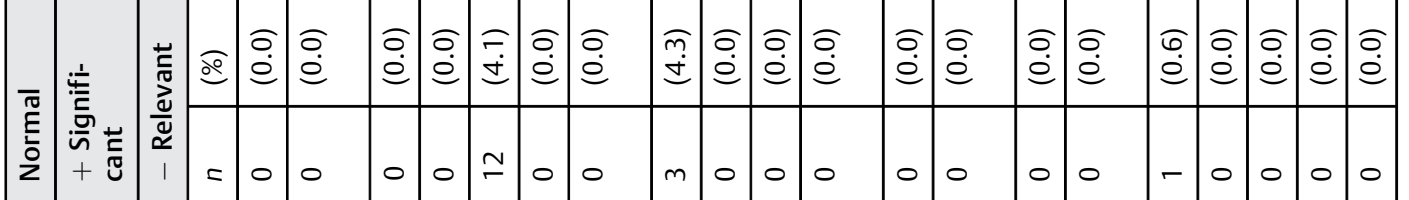

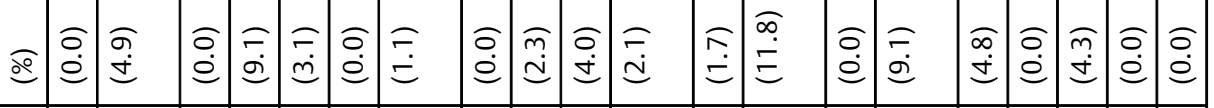

\begin{tabular}{|c|c|c|c|c|c|c|c|c|c|c|c|c|c|c|c|c|c|c|c|c|c|c|c|}
\hline & 节 & & $=$ & 0 & $\sim$ & 0 & - & $\sigma$ & 0 & - & 0 & $\nabla$ & - & - & $m$ & $\sim$ & 0 & - & $\infty$ & 0 & $m$ & 0 & 0 \\
\hline 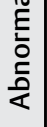 & $\begin{array}{l}\text { 意 } \\
\text { 离 } \\
+\end{array}$ & 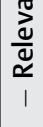 & Ð0 & $\mid \begin{array}{l}\bar{\partial} \\
\dot{0} \\
0\end{array}$ & $\mid \begin{array}{l}\tilde{n} \\
\infty \\
\end{array}$ & 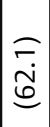 & 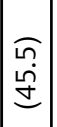 & $\begin{array}{c}\overline{=} \\
\dot{\dot{g}} \\
\dot{y}\end{array}$ & $\begin{array}{l}0 \\
\dot{0} \\
\text { in }\end{array}$ & 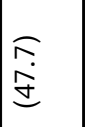 & $\left|\begin{array}{l}0 \\
\dot{j} \\
\dot{I}\end{array}\right|$ & 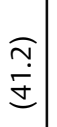 & 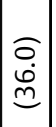 & 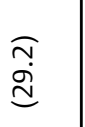 & 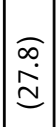 & $\begin{array}{l}\hat{\infty} \\
\stackrel{5}{E}\end{array}$ & $\mid \begin{array}{c}o \\
\dot{d} \\
\grave{d}\end{array}$ & $\overline{\bar{g}}$ & & 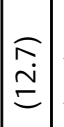 & $\stackrel{=}{=}$ & $\begin{array}{l}\bar{\Xi} \\
\bar{\Sigma}\end{array}$ & $\stackrel{\partial}{\circ}$ \\
\hline 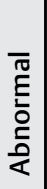 & 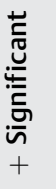 & 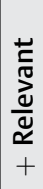 & $=$ & $\hat{m}$ & $\stackrel{ \pm}{\sim}$ & $\stackrel{\infty}{\sim}$ & in & $\stackrel{\tilde{m}}{\square}$ & $\wedge$ & $\mathcal{F}$ & Nิ & 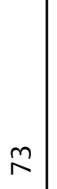 & a & $\stackrel{\nabla}{\square}$ & g & N & - & - & $\stackrel{m}{\sim}$ & 1 & Ln & - & 0 \\
\hline & 苞 & & తి & $\mid$\begin{tabular}{|c}
$\bar{\theta}$ \\
$\dot{0}$ \\
0
\end{tabular} & $\begin{array}{l}\widehat{\sigma} \\
\hat{\tilde{e}}\end{array}$ & $\begin{array}{l}\overrightarrow{\bar{v}} \\
\overrightarrow{\underline{v}}\end{array}$ & 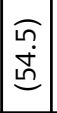 & $\begin{array}{l}\underset{\widehat{N}}{\sim} \\
\hat{n}\end{array}$ & 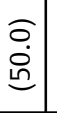 & $\begin{array}{l}\widehat{\sigma} \\
\infty \\
\stackrel{\infty}{ \pm}\end{array}$ & $\mid \begin{array}{l}\hat{f} \\
\dot{b} \\
\dot{y}\end{array}$ & $\begin{array}{l}\widetilde{n} \\
m \\
\tilde{\Xi}\end{array}$ & $\mid \begin{array}{c}o \\
0 \\
\dot{d} \\
\end{array}$ & $\frac{\bar{m}}{\frac{m}{m}}$ & 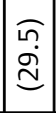 & 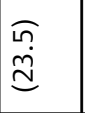 & 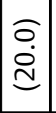 & $\begin{array}{l}\widehat{\widetilde{n}} \\
\stackrel{\infty}{=}\end{array}$ & $\begin{array}{c}\hat{m} \\
\stackrel{n}{=}\end{array}$ & 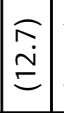 & 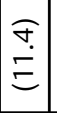 & $\begin{array}{l}\bar{C} \\
\bar{\Xi} \\
\bar{E}\end{array}$ & $\stackrel{\partial}{\dot{e}}$ \\
\hline & $\begin{array}{r}\bar{n} \\
+\end{array}$ & & $=$ & $\hat{m}$ & 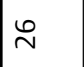 & $\stackrel{\infty}{-}$ & 0 & 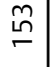 & $\wedge$ & $\stackrel{m}{\forall}$ & $\tilde{m}$ & $\curvearrowright$ & $\circ$ & $\stackrel{\text { Ln }}{\leftarrow}$ & กิ & $\nabla$ & - & $\sim$ & $\approx$ & $n$ & $\infty$ & - & 0 \\
\hline & & & 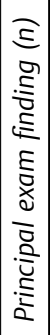 & 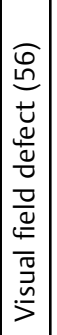 & 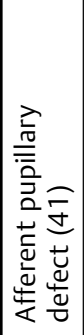 & 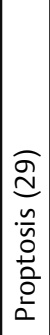 & 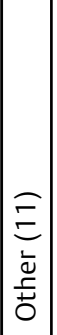 & 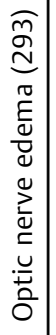 & 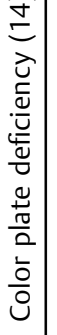 & 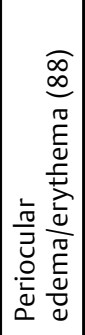 & 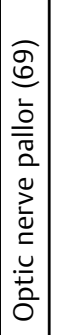 & 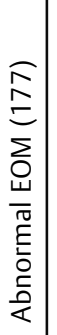 & 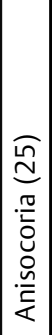 & 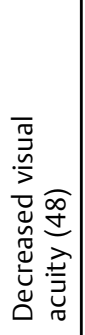 & 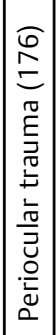 & 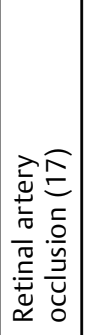 & 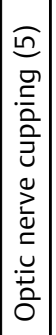 & 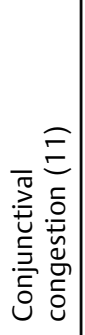 & 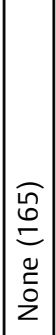 & 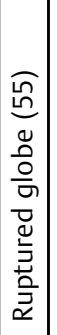 & 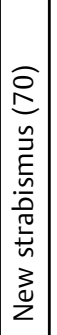 & 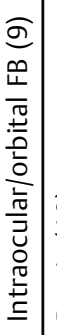 & $\begin{array}{l} \\
0 \\
0 \\
0 \\
\frac{n}{0} \\
0 \\
0 \\
0\end{array}$ \\
\hline
\end{tabular}




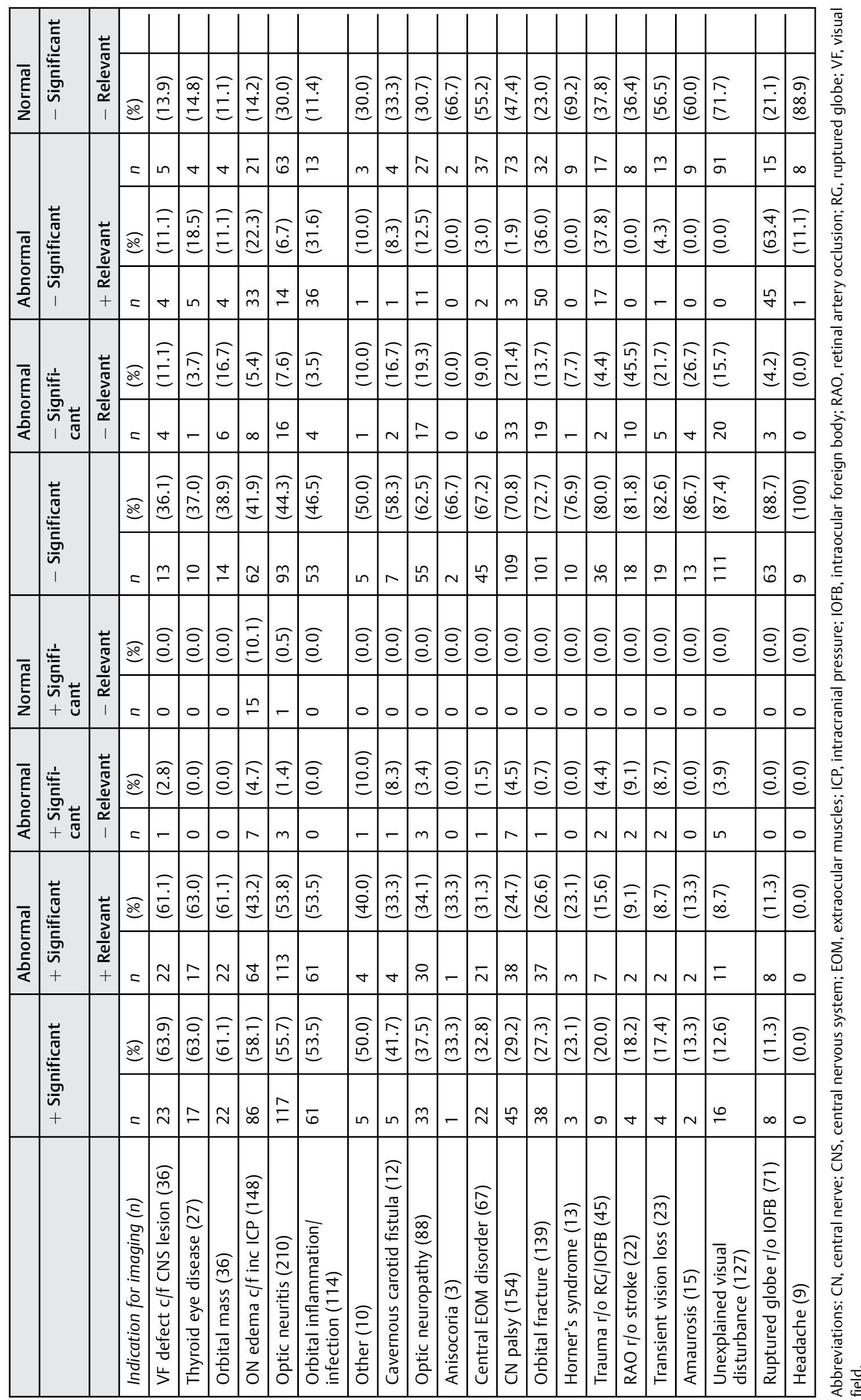


Table 4 Cost of neuroimaging by study

\begin{tabular}{|c|c|}
\hline Imaging study & Cost \\
\hline CT head $w /$ contrast & $\$ 166.32$ \\
\hline CT head $w / 0$ contrast & $\$ 118.08$ \\
\hline $\mathrm{CT}$ head $\mathrm{w} /$ and $\mathrm{w} / \mathrm{o}$ contrast & $\$ 195.12$ \\
\hline CT maxillofacial $\mathrm{w} /$ contrast & $\$ 170.64$ \\
\hline CT maxillofacial w/o contrast & $\$ 141.84$ \\
\hline CT orbits $w /$ contrast & $\$ 280.80$ \\
\hline CT orbits w/o contrast & $\$ 237.24$ \\
\hline CT orbits $w /$ and $w / o$ contrast & $\$ 306.00$ \\
\hline CTA head $w /$ and $w / o$ contrast & $\$ 298.44$ \\
\hline CTA neck $w /$ and $w / o$ contrast & $\$ 297.72$ \\
\hline MRI brain w/o contrast & $\$ 235.80$ \\
\hline MRI brain $w /$ and $w / o$ contrast & $\$ 385.56$ \\
\hline MRI orbit w/o contrast & $\$ 276.48$ \\
\hline MRI orbit w/ and w/o contrast & $\$ 411.84$ \\
\hline MRA head w/o contrast & $\$ 331.20$ \\
\hline MRA head w/ contrast & $\$ 334.08$ \\
\hline MRA head $w /$ and $w / o$ contrast & $\$ 493.19$ \\
\hline MRA neck w/o contrast & $\$ 366.84$ \\
\hline MRA neck w/ contrast & $\$ 335.88$ \\
\hline MRA neck $w /$ and $w / o$ contrast & $\$ 512.63$ \\
\hline
\end{tabular}

Abbreviations: CT, computed tomography; CTA, computed tomography angiography; MRA, magnetic resonance angiography; MRI, magnetic resonance imaging; $w$, with; w/o, without.

Note: The cost of imaging was determined using the Physician Fee Schedule Search found at the Centers of Medicare and Medicaid Services Web site and includes both the professional and technical components.

that prompted the physician to admit for lumbar puncture or begin empiric treatment with oral acetazolamide.

Eight hundred and fifty patients $(62.0 \%)$ had scans performed that were nonsignificant. Most of these patients' scans were normal (459, 33.5\%), without significant or relevant findings. Abnormal, nonsignificant, but relevant findings were found in 228 patients (16.6\%). An example from this outcome group included a patient with a clinically apparent ruptured globe, confirmed on imaging without additional findings. Finally, there were 163 patients (11.9\%) with abnormal findings that were nonsignificant and nonrelevant. Examples in this outcome group include multiple patients with nonspecific white matter changes of the brain or chronic orbital fractures not associated with their ER presentation.

Patients with significant imaging findings were more likely to be younger ( 44.7 vs. 48.4 years, $p=0.0006$, Rank-sum) and have worse VA (VA $20 / 68$ vs. $20 / 56, p=0.015$, Rank-sum). The odds of imaging resulting in a significant finding were higher (OR 1.57, 95\% CI 1.22-2.03, $p=0.001$, Chi-square test) when MRI only imaging $(n=880)$ was performed, compared with cases where CT imaging was utilized ( $n=491$ ).

The results of the subgroup analysis by patient reported CC can be found in -Table 1. Blurred vision $(n=395)$ was the most common symptom reported by patients who underwent imaging, followed by ocular and orbital trauma $(n=223)$, and double vision $(n=214)$. The highest yield CCs were periocular swelling (58.0\%), bulging eye(s) (57.1\%), and blurred vision (51.6\%). The lowest yield CCs were isolated anisocoria (22.2\%), visual disturbance (17.3\%), and patients who reported no complaint (15.8\%).

The subgroup analysis by principal examination finding can be found in -Table 2. The most common principal examination findings that underwent neuroimaging were optic nerve edema $(n=293)$, abnormal extraocular motility $(n=177)$, and periocular trauma $(n=176)$. The highest yield principal examination findings were visual field defect (66.1\%), afferent pupillary defector AFD (63.4\%), and proptosis $(62.1 \%)$. The lowest yield principal examination findings were ruptured globe (12.7\%), new onset strabismus (11.4\%), intraocular or intraorbital foreign body (11.1\%), and isolated ptosis $(0.0 \%)$.

The results of the subgroup analysis by indication for imaging can be found in - Table 3 . The most common indications for imaging were optic neuritis $(n=210)$, cranial nerve palsy $(n=154)$, and optic nerve edema concerning for increased intracranial pressure $(n=148)$. The highest yield indications for imaging were visual field defect concerning for a central nervous system (CNS) lesions (63.9\%), thyroid eye disease (TED) (63.0\%), and orbital mass (61.1\%). The lowest yield indications for imaging were amaurosis (13.3\%), unexplained visual disturbance (12.6\%), rule out intraocular foreign body (IOFB) after ruptured globe (11.3\%), and headache (0.0\%).

The cost of each imaging study can be found in - Table 4 . The total cost of imaging all 1,371 patients was $\$ 656,078.34$. The average cost of imaging per patient was $\$ 478.54$. The average cost of imaging per significant finding was $\$ 523.68$, $\$ 72.81$ more than the average cost per nonsignificant findings ( $p<0.0001$, rank sum). The average cost of imaging the abnormal, significant, and nonrelevant group was $\$ 598.65$,

Table 5 The yield of imaging by significant and relevant findings $(n=1,371)$

\begin{tabular}{|l|l|l|l|l|l|l|}
\hline & Abnormal & Abnormal & Normal & Abnormal & Abnormal & Normal \\
\hline & + Significant & + Significant & + Significant & - Significant & - Significant & - Significant \\
\hline & + Relevant & - Relevant & - Relevant & - Relevant & + Relevant & - Relevant \\
\hline Number of patients & 469 & 36 & 16 & 163 & 228 & 459 \\
\hline$(\%)$ & $(34.2 \%)$ & $(2.6 \%)$ & $(1.2 \%)$ & $(11.9 \%)$ & $(16.6 \%)$ & $(33.5 \%)$ \\
\hline $95 \%$ Confidence interval & $30.9-37.7 \%$ & $1.7-4.0 \%$ & $0.6-2.2 \%$ & $9.8-14.4 \%$ & $14.2-19.5 \%$ & $30.2-36.9 \%$ \\
\hline
\end{tabular}


Table 6 The cost of imaging by significant and relevant findings

\begin{tabular}{|l|l|l|l|l|l|l|}
\hline & Abnormal & Abnormal & Normal & Abnormal & Abnormal & Normal \\
\hline & + Significant & + Significant & + Significant & - Significant & - Significant & - Significant \\
\hline & + Relevant & - Relevant & - Relevant & - Relevant & + Relevant & - Relevant \\
\hline Number of patients & 469 & 36 & 16 & 163 & 228 & 459 \\
\hline Mean \# of images/patient & 2.07 & 2.39 & 2.44 & 2.04 & 1.54 & 2.01 \\
\hline Mean cost & $\$ 515.70$ & $\$ 598.65$ & $\$ 588.96$ & $\$ 462.69$ & $\$ 375.14$ & $\$ 484.30$ \\
\hline (SD) & $(\$ 269.75)$ & $(\$ 304.73)$ & $(\$ 280.78)$ & $(\$ 248.06)$ & $(\$ 223.89)$ & $(\$ 247.39)$ \\
\hline
\end{tabular}

Abbreviation: SD, standard deviation.

statistically more compared with the other outcome groups $(p<0.0001$, Kruskal-Wallis; - Table 6).

\section{Discussion}

Diagnostic imaging is an invaluable tool necessary to evaluate many ophthalmic diseases, both to establish a diagnosis and to eliminate the possibility of sight and life-threatening diseases in patients with unclear clinical presentations. In our study, neuroimaging was found to be significant, resulting in a change in management, in $38.0 \%$ of patients. Neuroimaging resulted in a change in management and was related to the patient's CC or examination findings in $34.2 \%$ of cases (significant and relevant). Our yield is similar to two smaller studies ( $n=157$ and $n=168$ ) which assessed the yield of imaging in outpatient neuroophthalmology offices. ${ }^{10,11}$ Mehta et al reported significant findings in $31.3 \%$ of outpatient neuroimaging tests, while significant and relevant findings were seen in $28.9 \% .{ }^{10}$ Pradhan et al reported significant findings in $36.1 \%$ of images, with both significant and relevant findings seen in $32.4 \%{ }^{11}$

The overall cost of imaging all 1,371 patients was $\$ 656,078.34$. The cost per clinically significant finding in our study was $\$ 523.68$. The cost per clinically significant and relevant finding was $\$ 515.70$, less than the $\$ 1,764.19$ per finding reported by Mehta et al who used the reimbursement rates from $2011 .^{10}$ The overall cost effectiveness in our study also compares favorably to other specialties. Jordan et al reported the cost per significant finding in the work-up of chronic headache to be $\$ 34,535$, while Liu et al reported the cost per significant finding in the work-up of vocal cord dysfunction to be $\$ 2,304 .{ }^{22,23}$ Our decreased cost is likely multifactorial and related to the decreasing cost of technology, increased number of imaging machines, and decreasing reimbursement.

Beyond the cost of performing the diagnostic imaging on our healthcare system, we must consider the human and financial cost if a diagnosis is missed. The Institute of Medicine has estimated that approximately 12 million people in the United States experience some form of diagnostic error/delay in their medical evaluation, resulting in $10 \%$ of patient deaths and 6 to $17 \%$ of hospital adverse events. ${ }^{24}$ The Institute of Medicine goes on to estimate the national cost of inefficiently delivered care due to diagnostic error, mistakes, and the subsequent preventable complications to be $\$ 130$ billion annually. ${ }^{25}$ Importantly, we must remember that the more significant cost of misdiagnosis falls upon the patients and their families in the form of lifelong care and lost income due to permanent disabilities or death.

The choice of neuroimaging modality (MRI vs. CT) is specific to the patient's presentation, suspected pathology, and comorbidities (e.g., presence of an MRI incompatible pacemaker). We found the odds of a significant finding were 57\% higher when MRI only imaging was performed, compared with cases where CT imaging was utilized. We suspect that the lower yield may be due to the physician's low threshold to utilize CT imaging as a screening modality in the settings of orbital trauma, penetrating trauma to assess for a ruptured globe, and to rule out IOFB in a ruptured globe. ${ }^{19}$ Unlike MRI imaging, CT imaging is relatively less expensive (-Table 5) and is shorter test in duration, making it a more practical screening instrument.

In our subgroup analysis by CC, the extraocular findings of periocular swelling and bulging eye(s) had the highest diagnostic yield (58.1 and 57.1\%, respectively). These findings were in alignment with Mehta et al who found the orbital "complaint" of proptosis was associated with the highest yield on imaging. ${ }^{10}$ Patients who did not report a CC (i.e., "none") and patients who described a visual disturbance were the lowest yield CCs (15.8 and $17.3 \%$, respectively). The patients who reported no CC were all asymptomatic at the time of presentation and were referred in by providers for concerning exam findings. The three patients who reported no CC and had significant findings on imaging, all had a relevant exam finding of optic nerve edema. Imaging was consistent with the diagnosis of optic neuritis in two patients and idiopathic intracranial hypertension in the third. Although symptom driven evaluations are at the core of the ophthalmologic assessment, we cannot be dismissive of patients without complaints or with abstract complaints, especially if their history or exam is suspicious for pathology.

Subgroup analysis by principal examination finding revealed a high diagnostic yield in patients with a visual field defect on confrontation (66.1\%), an $\operatorname{APD}(63.4 \%)$, and proptosis (62.1\%). This is in alignment with Mehta et al and reinforces the importance of close ophthalmic examination, paying attention for these findings. ${ }^{10}$ The lowest yield examination findings were isolated ptosis (0.0\%), ruptured globe with an IOFB (11.1\%), strabismus (11.4\%), and ruptured globe (12.7\%). The examination findings of isolated ptosis and strabismus were of similar low yield in the series by Mehta et al ( 0.0 and $0.0 \%$ significant, 
respectively). This suggests that a finding of isolated ptosis should seldomly warrant imaging unless there is strong suspicion for a structural lesion on complete neurological examination and review of systems. In contrast, it is always recommended to acquire $\mathrm{CT}$ imaging for the examination findings of ruptured globe with or without a foreign body as missing an IOFB is clinically devastating. ${ }^{19}$ of the 55 patients who underwent imaging for a ruptured globe an IOFB was found in seven (12.7\%) necessitating removal. Of the nine patients who had a clinically apparent ruptured globe with an IOFB, one patient (11.1\%) had a second, clinically undetected, orbital foreign body. Our data also support the imaging of patients who are not overtly clinically ruptured, but give a history of penetrating, explosive, projectile, or blunt force trauma directly to the eye. In our study, 7 of 45 patients (15.6\%) with such history were found to have occult ruptured globes on imaging requiring emergent operating room repair. Finally, a normal ophthalmic examination was associated with significant findings in $13.3 \%$ of the patients. This result should be approached with caution and should remind the ophthalmologist that a normal exam can conceal significant pathology.

Subgroup analysis by indication revealed a high diagnostic yield for a visual field defect concerning for a CNS lesion (63.9\%), TED (63.0\%), and orbital mass (61.1\%). Similarly, Mehta et al had a high diagnostic yield for TED (70.0\%) and Pradhan et al had a high diagnostic yield for extraocular orbital indications (68.7\%). ${ }^{10,11}$ The lowest yield indications with imaging in our series were amaurosis (13.3\%), unexplained visual disturbance (12.6\%), rule out an IOFB in a known ruptured globe (11.3\%), and headache $(0.0 \%)$. Headache was similarly the lowest yield indication in the study by Mehta et al (0.0\%). Jordan et $\mathrm{al}^{26}$ andWeingarten et $\mathrm{al}^{22}$ have both published large studies ( $n=1,233$ and $n=863$, respectively) looking at the yield of MRI imaging in patients with isolated headaches and found significant findings in only 0.0 to $1.5 \%$ of patients. This suggests that a headache without any additional neurologic or ophthalmic findings rarely warrants imaging. However, what if this headache represents an early symptom of an intracranial mass or aneurysm? This misdiagnosis certainly carries a human and financial cost that is more than the cost of the imaging. In a similar vein, the American Heart Association and the American Stroke Association have advocated for urgent MRI imaging of all patients who present with a transient ischemic attack (including amaurosis) as 10 to $15 \%$ of patients will have a stroke within 3 months, with half of those occurring within the first 48 hours. $^{27}$ Across multiple studies looking at MRI results in patients with transient ischemic attacks, MRI has shown at least one area of acute diffusion restriction in 25 to $67 \%$ of cases and at least onearea of infarct (acute or chronic) in 46 to $81 \%$ of the cases. ${ }^{27}$ We strongly support the neurological work-up of patients who present with amaurosis, including MRI of the brain. Regardless of the relatively low diagnostic yield for some indications, a resounding theme is that missing a potential imaging finding in patients with concerning history or examination findings may lead to devastating consequences for both the patient and the physician.

In this study we sought to determine the diagnostic yield and cost of imaging for ophthalmic conditions in an ER setting to help guide ophthalmologists in making costeffective imaging decisions. However, we acknowledge that there are limitations to this study. This study was designed to be exploratory in nature, to help set the foundation for larger, prospective, more detailed work. Although patients may present independently, our dedicated eye ER serves as an urgent or emergent tertiary referral center and our patient population is likely not representative of the general population. In contrast, it is also possible that referring providers will not send their patients for evaluation if they obtain imaging and make a diagnosis independently. Instead, they may opt to refer the patients directly to an appropriate subspecialist, leading to an underestimation of diagnostic yield. We also chose to include incidentally found pathology and normal imaging results that changed management (i.e., idiopathic intracranial hypertension) into our diagnostic yield. This decision was done to reflect a more real-world yield of imaging. We have reported the significant and relevant findings alongside the total significant findings for comparison. Finally, we calculated cost of imaging using only the billed CPT codes. This may underestimate the total theoretical cost to the hospital system but does give a better real-world representation of the cost of imaging.

In conclusion, neuroimaging for urgent and emergent ocular conditions provides useful diagnostic data that may change patient management at cost to the healthcare system. It is important for the ophthalmologist to be aware of the specific CCs, examination findings, and indications that are of particularly high and low yield. Finally, although imaging produces expenditures to our healthcare systems, we must not forget the substantial cost of misdiagnosis.

\section{Meeting Presentations}

American Academy of Ophthalmology 2019, San Francisco, CA, Podium Presentation.

\section{Funding/Support}

J. D. D. is a 2020-2021 Heed Fellow supported by the Heed Ophthalmic Foundation.

\section{Conflict of Interest}

None of the authors have disclosures related to the submitted work. R.C.S has the following disclosures outside of the submitted work: grants and speakers bureau for Biogen, reading center for clinical trials for Roche, speakers bureau for Mallinckrodt, reading center for clinical trials for Clene Nanomedicine, reading center for clinical trials for Medtronic, reading center for clinical trials for GenSight Biologics, advisory board for Celgene Bristol Meyers, data and safety monitoring committee for ProQR.

\section{References}

1 Dillon EC, Sergott RC, Savino PJ, Bosley TM. Diagnostic management by gatekeepers is not cost effective for neuro-ophthalmology. Ophthalmology 1994;101(09):1627-1630 
2 Kocher KE, Meurer WJ, Fazel R, Scott PA, Krumholz HM, Nallamothu BK. National trends in use of computed tomography in the emergency department. Ann Emerg Med 2011;58(05):452-462.e3

3 Larson DB, Johnson LW, Schnell BM, Salisbury SR, Forman HP. National trends in CT use in the emergency department: 19952007. Radiology 2011;258(01):164-173

4 Broder J, Warshauer DM. Increasing utilization of computed tomography in the adult emergency department, 2000-2005. Emerg Radiol 2006;13(01):25-30

5 Lee J, Kirschner J, Pawa S, Wiener DE, Newman DH, Shah K. Computed tomography use in the adult emergency department of an academic urban hospital from 2001 to 2007. Ann Emerg Med 2010;56(06):591-596

6 Korley FK, Pham JC, Kirsch TD. Use of advanced radiology during visits to US emergency departments for injury-related conditions, 1998-2007. JAMA 2010;304(13):1465-1471

7 Raja AS, Ip IK, Sodickson AD, et al. Radiology utilization in the emergency department: trends of the past 2 decades. AJR Am J Roentgenol 2014;203(02):355-360

8 Yoon P, Steiner I, Reinhardt G. Analysis of factors influencing length of stay in the emergency department. CJEM 2003;5(03):155-161

9 Chou KL, Galetta SL, Liu GT, et al. Acute ocular motor mononeuropathies: prospective study of the roles of neuroimaging and clinical assessment. J Neurol Sci 2004;219(1-2):35-39

10 Mehta S, Loevner LA, Mikityansky I, et al. The diagnostic and economic yield of neuroimaging in neuro-ophthalmology. J Neuroophthalmol 2012;32(02):139-144

11 Pradhan E, Bhandari S, Ghosh YK. The indications for and the diagnostic yield of imaging in neuro-ophthalmic and orbital disorders. Nepal J Ophthalmol 2015;7(14):159-163

12 Kupersmith MJ, Alban T, Zeiffer B, Lefton D. Contrast-enhanced MRI in acute optic neuritis: relationship to visual performance. Brain 2002;125(Pt 4):812-822

13 Lavin P, Patrylo M, Hollar M, Espaillat KB, Kirshner H, Schrag M. Stroke risk and risk factors in patients with central retinal artery occlusion. Am J Ophthalmol 2018;196:96-100

14 Murchison AP, Gilbert ME, Savino PJ. Neuroimaging and acute ocular motor mononeuropathies: a prospective study. Arch Ophthalmol 2011;129(03):301-305

15 Tamhankar MA, Biousse V, Ying G-S, et al. Isolated third, fourth, and sixth cranial nerve palsies from presumed microvascular versus other causes: a prospective study. Ophthalmology 2013; 120(11):2264-2269

16 Lee AG, Brazis PW, Garrity JA, White M. Imaging for neuro-ophthalmic and orbital disease. Am J Ophthalmol 2004;138(05):852-862
17 Lee AG, Johnson MC, Policeni BA, Smoker WR. Imaging for neuroophthalmic and orbital disease-a review. Clin Exp Ophthalmol 2009;37(01):30-53

18 Lakits A, Prokesch R, Scholda C, Bankier A. Orbital helical computed tomography in the diagnosis and management of eye trauma. Ophthalmology 1999;106(12):2330-2335

19 Yuan W-H, Hsu H-C, Cheng H-C, et al. CT of globe rupture: analysis and frequency of findings. AJR Am J Roentgenol 2014;202(05): $1100-1107$

20 Saeed A, Cassidy L, Malone DE, Beatty S. Plain X-ray and computed tomography of the orbit in cases and suspected cases of intraocular foreign body. Eye (Lond) 2008;22(11):1373-1377

21 Centers for Medicare and Medicaid Services. The Physician Fee Schedule. Accessed July 2019. Available at: https://www.cms.gov/ apps/physician-fee-schedule/

22 Weingarten S, Kleinman M, Elperin L, Larson EB. The effectiveness of cerebral imaging in the diagnosis of chronic headache. Arch Intern Med 1992;152(12):2457-2462

23 Liu AY, Yousem DM, Chalian AA, Langlotz CP. Economic consequences of diagnostic imaging for vocal cord paralysis. Acad Radiol 2001;8(02):137-148

24 National Academies of Sciences, Engineering, and Medicine. Improving Diagnosis in Health Care. Washington, DC: The National Academies Press; 2015

25 Institute of Medicine. Best Care at Lower Cost: The Path to Continuously Learning Health Care in America. Washington, DC: The National Academies Press; 2013

26 Jordan JE, Ramirez GF, Bradley WG, Chen DY, Lightfoote JB, Song A. Economic and outcomes assessment of magnetic resonance imaging in the evaluation of headache. J Natl Med Assoc 2000;92 (12):573-578

27 Easton JD, Saver JL, Albers GW, et al; American Heart Association; American Stroke Association Stroke Council; Council on Cardiovascular Surgery and Anesthesia; Council on Cardiovascular Radiology and Intervention; Council on Cardiovascular Nursing; Interdisciplinary Council on Peripheral Vascular Disease. Definition and evaluation of transient ischemic attack: a scientific statement for healthcare professionals from the American Heart Association/American Stroke Association Stroke Council; Council on Cardiovascular Surgery and Anesthesia; Council on Cardiovascular Radiology and Intervention; Council on Cardiovascular Nursing; and the Interdisciplinary Council on Peripheral Vascular Disease. The American Academy of Neurology affirms the value of this statement as an educational tool for neurologists. Stroke 2009;40(06):2276-2293 